\title{
ESPECIES FORESTALES Y ARBUSTIVAS ASOCIADAS A MACONELLICOCCUS HIRSUTUS (GREEN) (HEMIPTERA: PSEUDOCOCCIDAE) EN EL NORTE DE NAYARIT, MÉXICO
}

\author{
NÉSTOR ISIORDIA-AQUINO, ${ }^{1}$ AgUSTÍN ROBLES-BERMÚDEZ, ${ }^{2}$ \\ OSWALdo GARCÍA-MARTÍNEZ, ${ }^{3}$ REFUGIO LOMELÍ-FLORES, ${ }^{4}$ RICARDo \\ FLORES-CANALES, ${ }^{2}$ J. ROBERTO GÓMEZ-AGUILAR ${ }^{2}$ y ROSALBA \\ ESPINO-ALVAREZ ${ }^{2}$ \\ ${ }^{1}$ Estudiante de Posgrado, área de Ciencias Biológico Agropecuarias y Pesqueras, Universidad \\ Autónoma de Nayarit. Tepic, Nayarit, CP 63190. México. < nisiordia@gmail.com> \\ ${ }^{2}$ Unidad Académica de Agricultura, Universidad Autónoma de Nayarit. Km. 9, carretera Tepic- \\ Compostela; Xalisco, Nayarit. CP 63780. México. \\ ${ }^{3}$ Departamento de Parasitología Agrícola, Universidad Autónoma Agraria Antonio Narro. Buenavista, \\ Saltillo, Coahuila. CP 25315. México \\ ${ }^{4}$ Instituto de Fitosanidad, Colegio de Postgraduados. Km. 36.5, carretera México-Texcoco, Montecillo, \\ Estado de México. CP 56230. México.
}

Isiordia-Aquino, N., A. Robles-Bermúdez, O. García-Martínez, R. Lomelí-Flores, R. FloresCanales, J. R. Gómez-Aguilar \& R. Espino-Alvarez. 2012. Especies forestales y arbustivas asociadas a Maconellicoccus hirsutus (Green) (Hemiptera: Pseudococcidae) en el norte de Nayarit, México. Acta Zoológica Mexicana (n. s.), 28(2): 414-426.

RESUMEN. La presencia de cochinilla rosada del hibisco (CRH), Maconellicoccus hirsutus (Green) (Hemiptera: Pseudococcidae), causa serios daños ecológicos y socioeconómicos en Nayarit, México, por lo que demanda la realización de estudios que contribuyan a establecer un programa de manejo de esta plaga, entre los cuales resalta el conocimiento de sus hospederos. Los objetivos del estudio fueron conocer en que especies forestales y arbustivas del norte del estado se presenta este insecto y cuáles son sus niveles de infestación. Durante 2009 se seleccionaron 9235 puntos de muestreo, incluyendo áreas marginales, urbana, agrícola, forestal y viveros en siete municipios del norte de Nayarit; también se inspeccionaron 37 especies vegetales como posibles hospederos. El insecto estuvo presente en 1070 de los puntos muestreados (11.6\%) y de ellos el 94\% presentó un nivel de infestación 1 (bajo), para el nivel 2 (medio) 5.6\% y para el 3 (alto) sólo el 0.4\%. Las áreas que presentaron las mayores infestaciones fueron: las marginales (69.3\%) y la urbana (24.9\%); los municipios más afectados fueron Tuxpan, Ruíz y Rosamorada, con infestaciones promedio de 35.2, 22.3 y 12.4\%, respectivamente. Con respecto a las plantas hospederas se registraron 24 especies, mismas que se encuentran incluidas en nueve familias botánicas, con predominio de las Leguminosae; cinco de las especies identificadas correspondieron a nuevos registros como hospederos de $\mathrm{CRH}$ en el mundo. Tres leguminosas resultaron ser las más abundantes y también de las más preferidas por CRH, Acacia cochliacantha Schlecht \& Cham fue la especie

Recibido: 28/11/2011; aceptado: 30/03/2012. 
predominante ya que se encontró en todos los municipios (30.9\% de infestación), seguida por Mimosa pigra L. (19.3\%) y Albizia lebbeck (L.) Benth (14.5\%).

Palabras clave: Plaga cuarentenaria, cochinilla rosada del hibisco, muestreo de insectos, infestación de plagas, hospederos.

Isiordia-Aquino, N., A. Robles-Bermúdez, O. García-Martínez, R. Lomelí-Flores, R. FloresCanales, J. R. Gómez-Aguilar \& R. Espino-Alvarez. 2012. Forestry and shrub species associated to Maconellicoccus hirsutus (Green) (Hemiptera: Pseudococcidae) in northern Nayarit, Mexico. Acta Zoológica Mexicana (n. s.), 28(2): 414-426.

ABSTRACT. The presence of pink hibiscus mealybug (PHM), Maconellicoccus hirsutus (Green) (Hemiptera: Pseudococcidae), causes environmental and socioeconomic damage in Nayarit, México. This pest demands studies in order to help develop a management program that includes the knowledge of their hosts. The objectives of this study were to determine the hosts of this pest, such as trees or shrubs, and to know the levels of infestation on them. In 2009 there were selected and sampled 9235 points that included areas such as marginal, urban, agricultural, forestry and nurseries in seven municipalities of northern Nayarit. Also there 37 species were checked as possible host. The insect was present in 1070 points (11.6\%), of them 94\% presented an infestation at level one (low), to level two 5.6\% (medium), and only $0.4 \%$ to level three (high). The most infested areas were the marginal $(69.3 \%)$ and urban (24.9\%); the municipalities with higher infestations were Tuxpan, Ruiz, and Rosamorada with an average of 35.2, 22.3, and 12.4\%, respectively. About host plants there were recorded 24 species, included in nine botanical families, predominantly those belonging to the family Leguminosae; five of the identified plant species corresponded to new reports with PHM presence in the world. The most abundant and preferred to PHM were three leguminous species; however, the plant species that was present in all municipalities and predominant was Acacia cochliacantha Schlecht \& Cham (30.9\% infestation), followed by Mimosa pigra L. (19.3\%) and Albizia lebbeck (L.) Benth (14.5\%).

Key words: Quarantine pest, pink hibiscus mealybug, insect sampling, pest infestation, host plant.

\section{INTRODUCCIÓN}

La cochinilla rosada del hibisco (CRH), Maconellicoccus hirsutus (Green) (Hemiptera: Pseudococcidae), es una plaga polífaga de mucha importancia económica (Sagarra \& Peterkin 1999, Padilla 2000); la especie fue descrita inicialmente en India, desde donde se ha extendido a otras regiones tropicales y subtropicales del mundo, así como en algunas templadas, por lo que se considera una especie cuarentenaria de importancia mundial (Cermeli et al. 2002, Hoy et al. 2002, DGSV-DPF 2008). Se alimenta de savia, preferentemente en Hibiscus rosa-sinensis L. y es una amenaza seria ya que causa pérdidas económicas a la agricultura e industria forestal y de invernaderos (Mani 1989, Kairo 1998, Meyerdirk et al. 2003); afecta entre 200 a 300 especies vegetales, entre las que se encuentran hortalizas, ornamentales y forestales comprendidas en 70 familias botánicas, además se le ha observado alimentarse de especies vegetales silvestres, arvenses o de bosques tropicales y subtropicales; las familias más afectadas son Leguminosae, Malvaceae y Moraceae (Mani 1989, Garland 1998, Sagarra \& Peterkin 1999, Padilla 2000, USDA 2001, Meyerdirk et al. 2003, Bogran \& Ludwig 2007, DGSV-DPF 2008, Echegoyén \& González-Hernández 2010). En relación a la 
detección de poblaciones, Echegoyén \& González-Hernández (2010) señalan que las encuestas de monitoreo de las poblaciones de la CRH son parte de las acciones que deben de establecerse al detectar la plaga, para caracterizar las poblaciones en áreas infestadas, conocer la incidencia cuantitativa y los cambios que se experimentan a través del tiempo, de acuerdo a parámetros ambientales. Los hospederos de la CRH, según Sagarra \& Peterkin (1999), se dividen en dos categorías: a) vegetales donde puede completar su desarrollo y b) aquellos sobre los que se alimentan y produce síntomas de daños, pero sin completar su ciclo biológico.

Para Nayarit, González-Hernández (2011) reporta la presencia de la CHR en los municipios de Acaponeta, Bahía de Banderas, Compostela, El Nayar, Huajicori, Rosamorada, Ruiz, San Blas, Santiago Ixcuintla, Tecuala, Tepic, Tuxpan, Xalisco y Amatlán de Cañas, en niveles de infestación bajos.

La CRH es un problema muy importante para los agricultores nayaritas; por ser de reciente introducción en el estado, existen pocos estudios científicos cuya información coadyuve a manejar de manera eficiente a la plaga. Conocer su comportamiento y especies hospederas es básico, razón por la cual esta investigación tuvo como objetivos conocer las especies forestales y arbustivas sobre las que se encuentra presente el insecto y la determinación de sus niveles de infestación dentro de las áreas marginales, urbana, agrícola, forestal y viveros en siete municipios del norte de Nayarit.

\section{MATERIALES Y METODOS}

Muestreo. Los muestreos para identificar especies vegetales forestales y arbustivas hospederas de CRH durante el periodo de estudio (enero-diciembre de 2009), se realizaron mediante colectas periódicas hasta cubrir la totalidad de especies vegetales en el norte del estado de Nayarit. Las áreas consideradas fueron: marginales (o no comerciales, ubicadas en zonas de traspatio y sobre caminos vecinales), urbanas, agrícolas, forestales y viveros, en los municipios de Acaponeta, Tecuala, Huajicori, Rosamorada, Tuxpan, Ruiz y Santiago Ixcuintla.

Identificación de especies vegetales hospederas. Para confirmar la presencia de $M$. hirsutus sobre la generalidad de especies vegetales hospederas consideradas en el estudio, se realizaron colectas y montajes del insecto en sus diferentes estadios de desarrollo, conforme a la metodología utilizada por SENASICA (2011), para lo que se consideró a plantas afectadas por sus síntomas visibles de daños como malformaciones severas en retoños y hojas, arrugamiento y detención de crecimiento en hojas, etc. (Francis 1995), además de corroborar la identidad del insecto plaga mediante verificación de características morfológicas externas, conforme a lo descrito por autores como William (1996), Miller (1999), Gullan (2000) y Meyerdirk et al. (2003). Para cada vegetal se colectaron hojas, tallos, flores y frutos, mismos que se prensaron y deshidrataron mediante la técnica estándar de herborización (Lot \& Chiang 1986, Maden 2004). La identificación taxonómica se realizó en la Unidad Académica de 
Agricultura de la Universidad Autónoma de Nayarit (UAN), con confirmación mediante cotejo de muestras con las del Herbario Nacional de la Universidad Nacional Autónoma de México (MEXU).

Determinación del nivel de infestación de CRH. Para determinar el nivel de infestación de la CRH en todas las especies vegetales dentro de cada área de muestreo, se seleccionaron y geocodificaron puntos o sitios de muestreo permanentes, en donde se revisaron cuatro brotes terminales de $5-10 \mathrm{~cm}$ de 5 a 10 plantas (DGSVDPF 2008). Para la inspección de las áreas marginales se revisaron puntos cada 500$1000 \mathrm{~m}$ sobre caminos, ríos y carreteras, conforme a la disponibilidad de hospederos preferidos por el insecto en cada municipio; estos muestreos se realizaron en forma semanal, para lo cual se contabilizó el total de especímenes de CRH en los estadios ninfales (primero, segundo y tercero) y adulto. Se utilizó el mismo procedimiento de conteo del insecto para las otras áreas, excepto que en las áreas urbanas los muestreos se realizaron en forma quincenal dentro de puntos establecidos conforme a la disponibilidad de hospederos. La inspección para las áreas agrícolas se hizo acorde a la fenología de los hospederos, el muestreo en cada unidad de producción no mayor de 1 ha, se hizo en zigzag con cinco árboles; ocho en unidades de $>1-2$ ha; 12 en unidades de >2-3 ha; 15 en unidades de >3-4 ha y 20 árboles en unidades mayores de 4 ha. En las áreas forestales se seleccionaron los cercos o lienzos de las parcelas y se muestrearon 12 puntos aleatoriamente, tres por cada lado de la parcela. En viveros el muestreo fue directo y se revisaron diversas plantas tomadas al azar. En todos los casos, para obtener el nivel de infestación de CRH en cada uno de los muestreos, se promedió el total de especímenes contabilizados dentro de cada una de las especies vegetales entre el número de brotes muestreados en los puntos o sitios, lo que permitió además obtener dichos promedios en forma mensual de acuerdo con la ubicación de cada una de las especies muestreadas a nivel municipal, al dividir el total de especímenes entre el número total de brotes terminales muestreados en el mes.

Para conocer el nivel de infestación se utilizó la escala establecida por la DGSVDPF (2008) y Suresh \& Chandra (2008), las que especifican que el nivel nulo corresponde a 0 cochinillas por brote; el nivel 1 ó bajo de 1-10 cochinillas por brote; el nivel 2 ó medio de 10-20 cochinillas por brote; nivel 3 ó alto con más de 20 cochinillas por brote. Se utilizó como herramienta la estadística descriptiva, lo que permitió determinar las frecuencias de la presencia del insecto.

\section{RESULTADOS}

Identificación de especies vegetales hospederas. De las 37 especies vegetales inspeccionadas como posibles hospederos, se logró determinar que 24 de ellas resultaron asociadas a CRH, estas plantas se encuentran ubicadas en nueve familias (Cuadro 1); en orden de importancia se indican a continuación: Leguminosae (13 especies), Moraceae (3), Asteraceae (2), Sterculiaceae (2), Euphorbiaceae (1) Meliaceae (1), 
Isiordia-Aquino et al. Especies forestales asociadas a Maconellicoccus en Nayarit

Cuadro 1. Especies forestales o arbustivas con presencia de $M$. hirsutus Green en el norte de Nayarit.

\begin{tabular}{|c|c|c|c|}
\hline Familia & Nombre científico & Nombre común & Municipio \\
\hline \multirow{2}{*}{$\begin{array}{l}\text { Asteraceae ( = } \\
\text { Compositae) }\end{array}$} & Tithonia diversifolia (Hemsl.) A. Gray & tacotillo & $1,2,3,4$ \\
\hline & Verbesina greenmanii Urban & tacote & $1,4,5$ \\
\hline Euphorbiaceae & Phyllantus elsiae Urb. & pimientillo & 4,5 \\
\hline \multirow{13}{*}{$\begin{array}{l}\text { Leguminosae ( = } \\
\text { Fabaceae) }\end{array}$} & Acacia acatlensis Benth. & parotilla & 4 \\
\hline & Acacia cochliacantha Schlecht. \& Cham & concha ( = huinol) & $1,2,3,4,5,6,7$ \\
\hline & Acacia farnesiana L. Willd. & Huizache & 7 \\
\hline & Acacia hindsii Benth & jarretadera & $1,3,4,5,7$ \\
\hline & Albizia lebbeck (L.) Benth & capiro & $1,2,4,5,7$ \\
\hline & Delonix regia (Bojer Ex Hook.) Raf. & tabachin & 4 \\
\hline & Enterolobium cyclocarpum (Jacq.) Griseb & $\begin{array}{l}\text { parota (= } \\
\text { huanacaztle) }\end{array}$ & $1,2,4,5,6,7$ \\
\hline & Glyciridia sepium (Jacq.) Kunth. Ex Walp & $\begin{array}{l}\text { catispa ( = } \\
\text { cacahuananchi) }\end{array}$ & 7 \\
\hline & Inga eriocarpa Benth. & $\begin{array}{l}\text { jiquinicuil ( = } \\
\text { cuajinicuil) }\end{array}$ & $1,2,3,6$ \\
\hline & Mimosa invisa Mart. Ex colla & sierrilla & 4,5 \\
\hline & Mimosa pigra L. & coatante & $1,2,4,5,6,7$ \\
\hline & Pithecellobium sp. & guamuchilillo & 6,7 \\
\hline & Prosopis laevigata (H. \& B.) Jonhston & mezquite & 1 \\
\hline Meliaceae & Cedrella odorata L. & cedro & 7 \\
\hline \multirow[t]{3}{*}{ Moraceae } & Ficus obtusifolia Kunth & chalata, salate & 6 \\
\hline & Ficus pertusa L.f. & camichin & 1 \\
\hline & Ficus sp. & higuera & 5 \\
\hline Solanaceae & Solanum umbellatum Mill. & tabaquillo & $2,4,5$ \\
\hline \multirow[t]{2}{*}{ Sterculiaceae } & Byttneria aculeata (Jacq.) Jacq. & rabo de iguana & $1,2,4,5$ \\
\hline & Guazuma ulmifolia Lam. & guásima & 7 \\
\hline Vervenaceae & Tectona grandis L. & teca & $1,4,6,7$ \\
\hline
\end{tabular}

1 Acaponeta; 2 Tecuala; 3 Huajicori; 4 Rosamorada; 5 Tuxpan; 6 Ruiz; 7 Santiago Ixc.

Solanaceae (1) y Verbenaceae (1). Por su nivel de infestación, A. cochliacantha fue el hospedero más importante, además de que estuvo presente todo el año y en todos los municipios (30.9\%), seguida por M. pigra y A. lebbeck (19.3 y 14.5\%, respectivamente); en las especies E. cyclocarpum (11.9\%) y G. sepium (6.7\%) CRH se presentó en menor grado(Cuadro 3). Con respecto a las 19 especies hospederas restantes, éstas 
se registraron sólo en 177 puntos (16.5\%). Las especies C. odorata, G. ulmifolia, $P$. laevigata, $T$. diversifolia y $V$. greenmani representan nuevos registros de especies vegetales afectadas por el insecto plaga en el mundo, todos en un nivel de infestación 1 (Echegoyéns \& González 2010, González-Hernández 2011, Meyerdirk et al. 2002, ScaleNet 2011).

Determinación de nivel de infestación y dispersión. Los promedios de la CRH obtenidos por brote en los 9235 puntos muestreados, reflejan que la plaga estuvo presente en 1070 (11.6\%), de éstos, y que en los 8165 restantes (88.4\%) no se observó infestación (Cuadro 2). El mayor número de puntos muestreados e infestados correspondió a los municipios de Rosamorada (2465: 169), Acaponeta (2461: 306) y Santiago Ixcuintla (2065: 194). En los municipios de Tuxpan, Ruíz y Rosamorada se registraron los mayores porcentajes de infestación, con el 35.2, 22.3 y 12.4\%, respectivamente. Del total de puntos con presencia de la CRH, el 94\% correspondió a un nivel 1 (bajo), seguido de los niveles 2 (medio) y 3 (alto), con el 5.6 y $0.4 \%$, respectivamente. En este aspecto, los municipios con mayor cantidad de puntos con presencia del insecto fueron Rosamorada (28.7\%), Santiago Ixcuintla (18.1\%) y Tuxpan (16.2\%). Finalmente, en lo que se refiere a las diversas áreas de muestreo, las afectaciones más fuertes ocurrieron en las áreas marginales (69.3\%) y urbana (24.9\%), con muy poca presencia en las áreas forestal y agrícola, y nula en viveros. A nivel municipio, por el grado de afectación en áreas de muestreo, se observaron las mismas dominancias, existiendo muy poca variación porcentual de los resultados entre dichos municipios.

Con respecto a su fenología, por el número de puntos monitoreados y con presencia de $\mathrm{CRH}$, la dominancia en la cantidad de puntos infestados se observó durante los meses de abril a agosto, mientras que en el periodo comprendido entre los meses de enero a marzo y noviembre- diciembre el número de puntos con presencia del insecto fue mucho menor (Figura 1).

\section{DISCUSIÓN}

Mani (1989), Garland (1998), Sagarra \& Peterkin (1999), Padilla (2000) y DGSVDPF (2008) han registrado la preferencia que CRH tiene por hospederos de las familias Leguminosae y Moraceae, observaciones que coinciden con nuestros resultados. Por otro lado, Chavan \& Kadam (1989), Murgueitio et al. (1999) y Goyoaga (2005), indican que entre las causas por las cuales las leguminosas son más susceptibles a infestaciones por $\mathrm{CRH}$, son sus altos contenidos de proteína y nitrógeno, así como un menor grado de fibra, lignina, fósforo, calcio y taninos; también es importante tomar en cuenta que en las regiones tropicales y subtropicales, este tipo de plantas tienen una alta proliferación debido a la temperatura y humedad óptima para ellas.

Para el caso de especies como A.cochliacantha, M. pigra y E. cyclocarpum, también existe concordancia con lo reportado por González-Hernández (2011), quien en 
Isiordia-Aquino et al. Especies forestales asociadas a Maconellicoccus en Nayarit

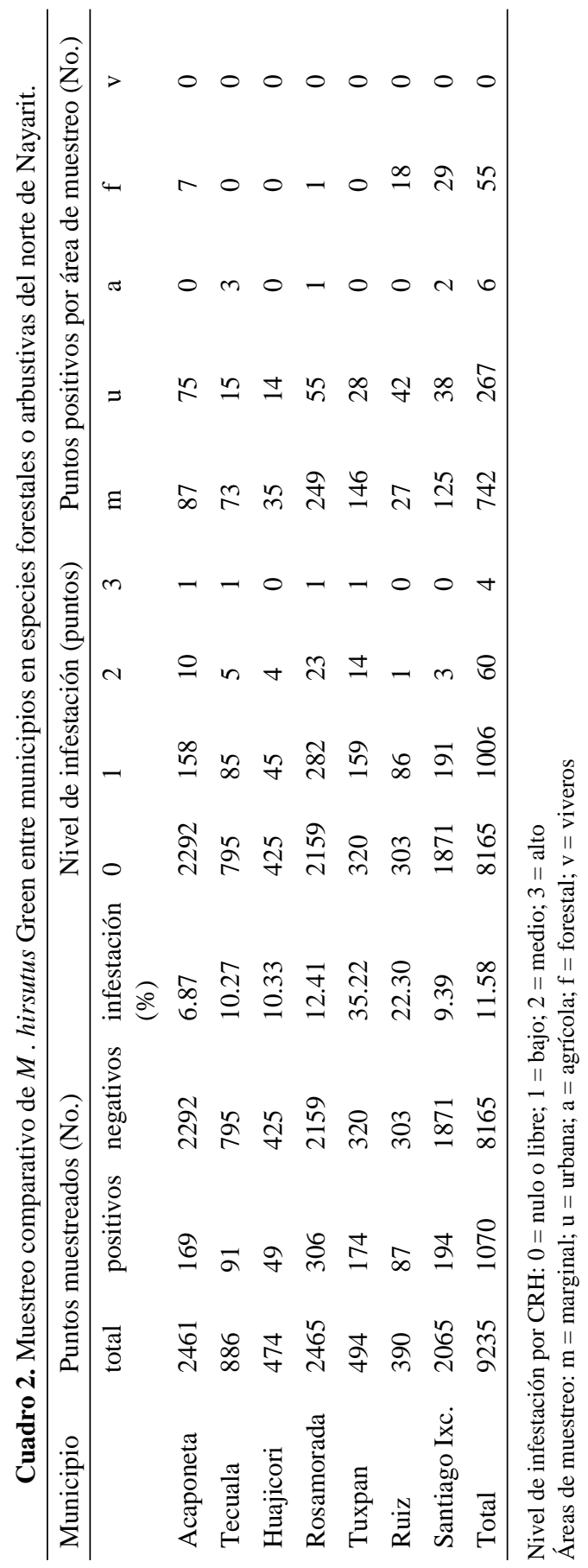


Acta Zool. Mex. (n.s.) 28(2) (2012)

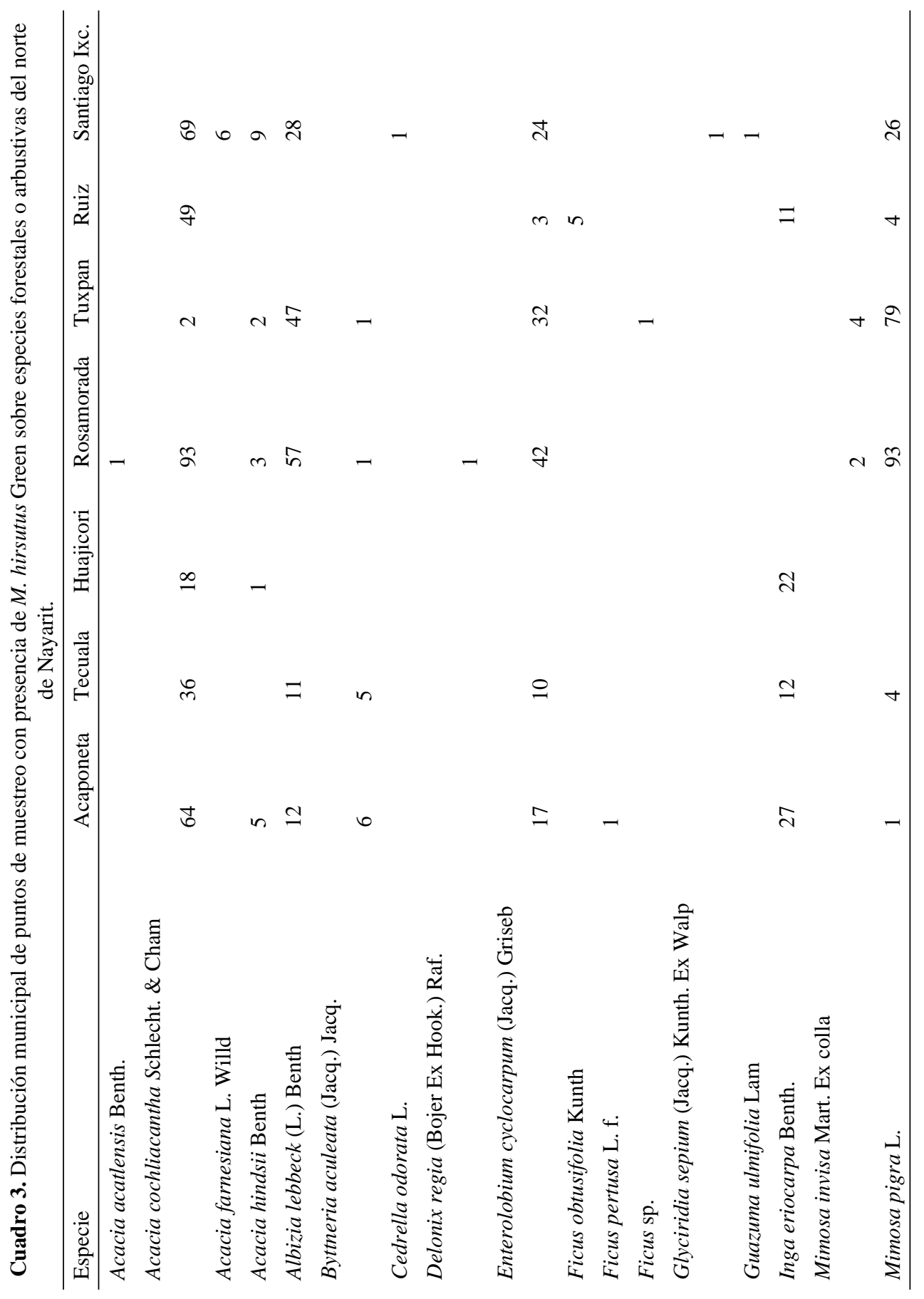


Isiordia-Aquino et al. Especies forestales asociadas a Maconellicoccus en Nayarit

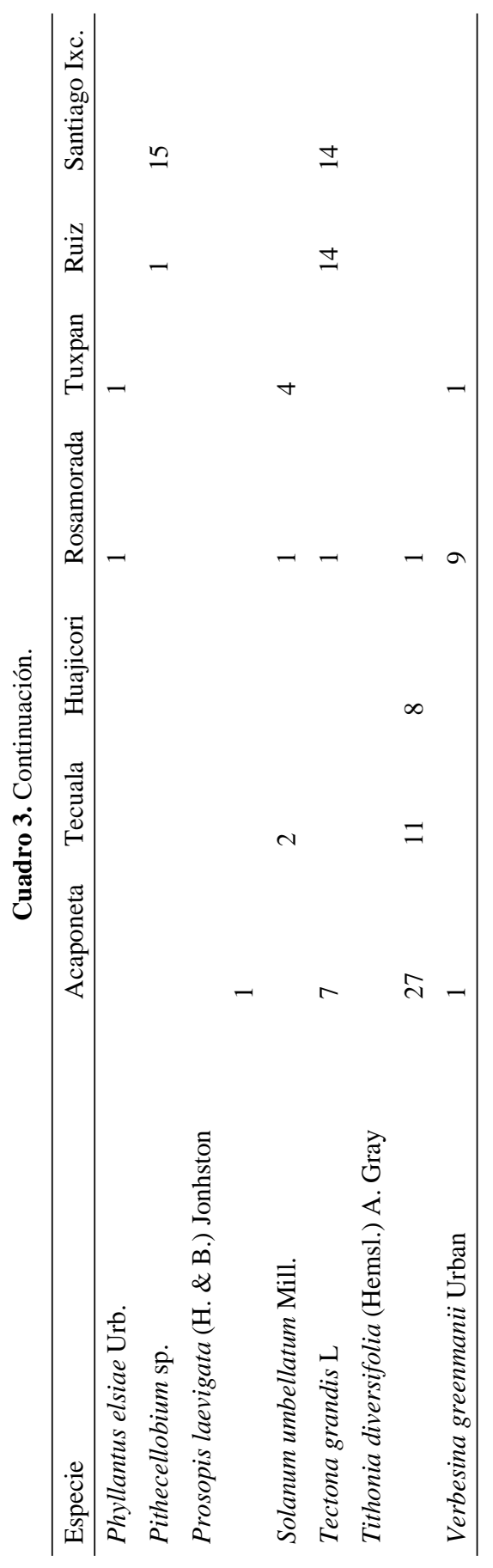




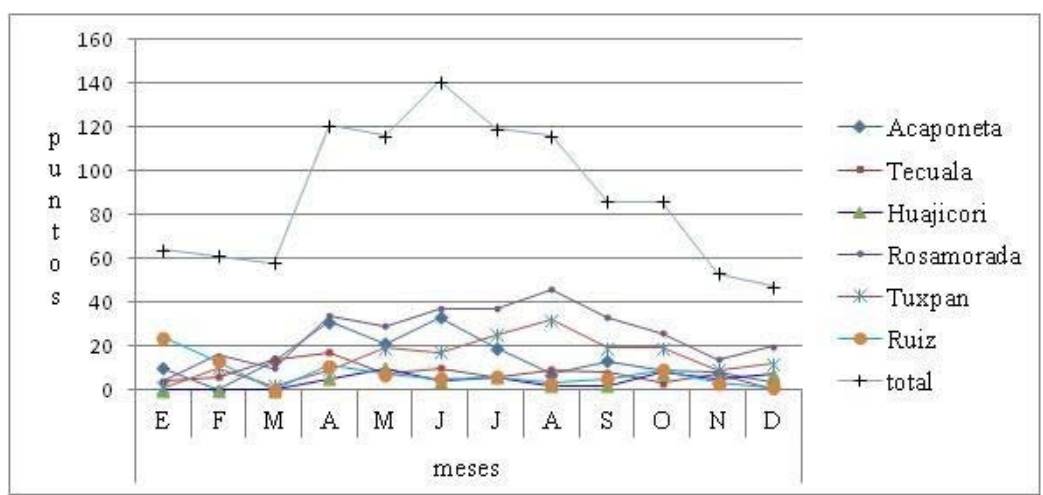

Figura 1. Distribución mensual de puntos de muestreo con presencia de $M$. hirsutus Green en especies forestales o arbustivas del norte de Nayarit.

una modificación a lo publicado por DGSV-DPF (2008), menciona que para la región de Bahía de Banderas (Nayarit y Jalisco) la CRH desarrolló fuertes infestaciones al inicio de su establecimiento en vegetación de áreas marginales, junto con otras especies como Senna hirsutta, Salix sp. y Schinus molle. En cuanto a la especie A. lebbeck, no obstante de ser considerada como hospedante secundario (CABI 2005), existen evidencias de haber sufrido serios daños por este insecto en Egipto (Mani 1989); de igual manera para T. grandis y G. sepium, también hay registros de ser muy susceptibles al insecto (Sagarra \& Peterkin 1999).

Los resultados de presencia y niveles de infestación concuerdan con los reportados por González-Hernández (2011) para el mismo año de estudio (2009) dentro de la región sur del mismo estado (Nayarit) y del estado de Jalisco en los cuales, no obstante que en los años 2004 y 2005 se detectaron altos niveles de infestación, la situación en 2008 y 2009 cambió drásticamente tanto en áreas agrícolas, como marginales y urbanas, donde los promedios en niveles poblacionales de la plaga se mantuvieron bajos, entre 0.4 (Jalisco) y 0.5 (Nayarit) cochinillas por brote, gracias a las acciones de manejo integrado implementadas desde su aparición, pero particularmente debido al impacto de los enemigos naturales (Anagyrus kamali Moursi y Cryptolaemus montrouzieri Mulsant) liberados periódicamente en la región.

La probable causa por la cual el número de puntos de muestreo con presencia del insecto disminuyó durante los meses de enero a marzo y noviembre-diciembre (Figura 1) se debió a las bajas temperaturas en esos periodos (Figura 2); este fenómeno ya fue registrado por Chong et al. (2008), quienes observaron que este factor fue determinante en la tasa de reproducción y longevidad de los diferentes estados de desarrollo del insecto, también registraron que la temperatura umbral mínima de desarrollo para las hembras fue de $14.5^{\circ} \mathrm{C}$ y para los machos de $14.3^{\circ} \mathrm{C}$; indicaron también que a la 
Isiordia-Aquino et al. Especies forestales asociadas a Maconellicoccus en Nayarit

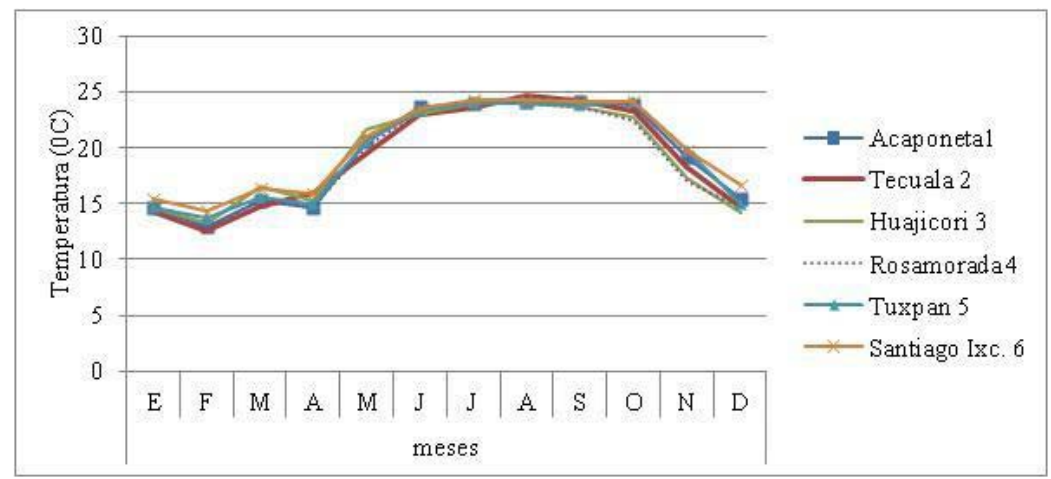

Figura 2. Promedios de temperatura mínima mensual en 2009 en el norte de Nayarit.

temperatura de $16{ }^{\circ} \mathrm{C}$ no hay eclosión de huevos, por lo que sugieren que dicho insecto sólo puede sobrevivir a temperaturas mayores de $15^{\circ} \mathrm{C}$. Del mismo modo Marcano et al. (2006) evaluaron el efecto de cinco temperaturas constantes sobre el tiempo de desarrollo, fecundidad y fertilidad de CRH en papa colombiana (Solanum phureja Juz. et Buk.) como alimento, no encontraron emergencias de ninfas a $15^{\circ} \mathrm{C}$. Por otro lado, si se toma en cuenta que más del $50 \%$ de puntos de muestreo con presencia de CRH se encontraron durante el periodo abril-agosto, quizás sea debió a las condiciones bióticas y abióticas favorables al desarrollo del insecto en la región, como lo es el alimento (plantas hospederas), temperaturas y humedad relativa, sin que al parecer se haya tenido algún impacto negativo por las precipitaciones ocurridas en los meses de julio y agosto. Al respecto Katke (2008) al realizar un trabajo sobre biología, manejo e incidencia estacional de CRH dentro del cultivo de vid en India, encontró que para el caso de ovisacos, ninfas y adultos detectó una correlación positiva y altamente significativa con temperaturas máximas y mínimas, así como una correlación negativa y altamente significativa con humedad relativa temprano y durante las tardes, lo mismo que de una correlación negativa no significativa con la lluvia.

Agradecimientos. Al Consejo Nacional de Ciencia y Tecnología (CONACYT) por el apoyo económico para la realización del estudio, mediante proyecto Nayarit 2007/C04/81795, así como al Comité Estatal de Sanidad Vegetal de Nayarit (CESAVENAY), por las facilidades de información proporcionadas, lo mismo que al personal del área de Biología que apoyó en la identificación de especies en la UAN.

\section{LITERATURA CITADA}

Bogran, C. E. \& S. Ludwig. 2007. Pink hibiscus mealybug, a new pest in Texas. Texas Cooperative Extension, The Texas A\&M University System E-454.

CABI. 2005. Crop Protection Compendium. Global Module. CAB International. United Kingdom. 
Cermeli, M., V. P. Morales, F. Godoy, R. Romero \& O. Cárdenas. 2002. Presencia de la cochinilla rosada de la cayena Maconellicoccus hirsutus (Green) (Hemíptera: Pseudococcidae) en Venezuela. Entomotrópica, 17: 103-105.

Chavan, J. K. \& S. S. Kadam. 1989. Protease inhibitors, pp. 123-133. In: D. K. Salunkhe \& S. S. Kadam (Eds). Handbook of World Food Legumes: Nutritional Chemistry, Processing Technology, and Utilization. Vol. 1. CRC Press, Boca Ratón, Florida, USA.

Chong, J. H., A. L. Roda \& C. M. Mannion. 2008. Life history of the mealybug, Maconellicoccus hirsutus (Hemiptera: Pseudococcidae), at constant temperatures. Environmental Entomology, 37: 323-332.

DGSV-DPF. 2008. Apéndice Técnico-Operativo de la Campaña contra cochinilla rosada del hibisco (Maconellicoccus hirsutus Green). Dirección General de Sanidad Vegetal, Servicio Nacional de Sanidad, Inocuidad y Calidad Agroalimentaria, Secretaría de Agricultura, Ganadería, Desarrollo Rural, Pesca y Alimentación (DGSV, DPF, SENASICA, SAGARPA). Versión 1. México, D.F. 36 pp.

Echegoyén, R. P. \& H. González H. 2010. Plan de Contingencia ante un Brote de Cochinilla Rosada del Hibisco (Maconellicoccus hirsutus) en un País de la Región del OIRSA. Organismo Internacional Regional de Sanidad Agropecuaria (OIRSA). San Salvador, El Salvador. 165 pp.

Francis, E. D. 1995. Paper on background and status of mealybug Maconellicoccus hirsutus in Granada. Ministry of Agriculture, Granada. 7 pp.

Garland, J. A. 1998. Pest Risk Assessment of the pink mealybug Maconellicoccus hirsutus (Green), with particular reference to Canadian greenhouses. PRA 96-21. Canadian Food Inspection Agency, Ottawa, Ca.

González-Hernández, H. 2011. Ficha Técnica Maconellicoccus hirsutus (Green) Cochinilla Rosada del Hibisco (CRH). Secretaría de Agricultura, Ganadería, Desarrollo Rural, Pesca y Alimentación, Servicio Nacional de Sanidad, Inocuidad y Calidad Agroalimentaria, Sistema Nacional de Vigilancia Epidemiológica Fitosanitaria (SAGARPA, SENASICA, SINAVEF). México, D.F. 32 p. Consulta: 14 de septiembre de 2011. En Línea: http://www.cesaveson.com/fckeditor/editor/filemanager/conectors/oispx/UserFiles/file/FT_CRH_2011.pdf.

Goyoaga, J. C. 2005. Estudio de factores no nutritivos en Vicia faba L. Influencia sobre su valor nutritivo. Tesis Doctor en Ciencias, Universidad Complutense de Madrid. 254 p.

Gullan, P. J. 2000. Identification of the inmature Instars of mealybugs (Hemiptera: Pseudococcidae) found on citrus in Australia. Australian Journal of Entomology, 39:160-166.

Hoy, M. A., A. Hamon \& R. Nguyen. 2002. Pink hibiscus mealybug, Maconellicoccus hirsutus (Green). EENY-029, University of Florida University IFAS Extensión. 6 p.

Kairo, M. T. K. 1998. Biology of the hibiscus (pink) mealybug, Maconellicoccus hirsutus (Green), Report for the Pink Hibiscus Mealybug Technology Transfer Workshop. USVI. 6 p.

Katke, M. 2008. Seasonal incidence, Biology and Management of grape mealy bug, Maconellicoccus hirsutus (Green). M.Sc. (Agri.) Thesis. Department of Agricultural Entomology College of Agriculture, Dharwad University of Agricultural Sciences. Dharwad, India. 89 pp.

Lot, A. \& F. Chiang. 1986. Manual de herbario. Consejo Nacional de la Flora de México A.C. México. $142 \mathrm{pp}$.

Maden, K. 2004. Plant Collection and Herbarium Techniques. Our Nature, 2: 53-57.

Mani, M. 1989. A rewiew of the pink mealybug Maconellicoccus hirsutus (Green). Insect Science and its Application, (10): 157-167.

Marcano, R., B. Nienstaedt, S. Longa \& T. Malpica. 2006. Efecto de la temperatura sobre el tiempo de desarrollo, fecundidad y fertilidad de la cochinilla rosada Maconellicoccus hirsutus (Green), (Hemiptera: Pseudococcidae). Entomotropica, 21 (1): 19-22. 
Isiordia-Aquino et al. Especies forestales asociadas a Maconellicoccus en Nayarit

Meyerdirk, D. E., R. Warkentin, B. Attavian, E. Gersabeck, A. Francis, M. Adams \& G. Francis. 2003. Manual del Proyecto para el Control Biológico de la Cochinilla Rosada del Hibisco. Traducción al español por IICA en acuerdo con el USDA. 2a. Edición. USDA-IICA. San José, Costa Rica. 194 p.

Miller, D. R. 1999. Identification of the Pink Hibiscus Mealybug, Maconellicoccus hirsutus (Green) (Hemiptera: Sternorrhyncha: Pseudococcidae). Insecta Mundi, 13: 189-203.

Murgueitio, R. E., M. M. Rosales \& M. E. Gómez. 1999. Agroforestería para la Producción Animal Sostenible. 1a. Edición. Fundación CIPAV. Cali, Colombia. 67 pp.

Padilla, M. R. 2000. Bioecología de la cochinilla rosada y su riesgo de ingreso en Honduras. Manejo Integrado de Plagas (Costa Rica), 57: 10-22.

ScaleNet. 2011. Catalogue Query Results. Maconellicoccus hirsutus (Green). Consulta 03 de marzo de 2012. En Línea: http://www.sel.barc.usda.gov/catalogs/pseudoco/Maconellicoccushirsutus.htm.

Sagarra, L. A. \& D. D. Peterkin. 1999. Invasion of the Caribbean by the hibiscus mealybug, Maconellicoccus hirsutus Green (Homóptera: Pseudococcidae). Phytoprotection, 80: 103-113.

SENASICA. 2011. Procedimiento para la Evaluación y Diagnóstico de Maconellicoccus hirsutus (Green) (Hemiptera: Pseudococcidae) y sus Enemigos Naturales en Laboratorio. Servicio Nacional de Sanidad, Inocuidad y Calidad Agroalimentaria, Centro Nacional de Referencia Fitosanitaria, Centro Nacional de Referencia de Control Biológico (SENASICA, CNPF, CNRCB). Versión 1. 29 pp.

Suresh, S. \& K. P. Chandra. 2008. Seasonal Incidence of economically important coccid pests in Tamil Nadu. pp. 285-291. In: M. Branco, J.C. Franco \& C.J. Hodgson (Eds). Proceedings of the XI International Symposium on Scale Insect Studies. Oeiras, Portugal.

USDA. 2001. Biological Control of Pink Hibiscus Mealybug Project Manual. 194 pp.

Williams, D. J. 1996. A brief account of the hibiscus mealybug Maconellicoccus hirsutus (Hemiptera: Pseudococcidae), a pest of agriculture and horticulture, with descriptions of two related species from sothern Asia. Bulletin of Entomological Research, 86:617-628. 